\title{
Quadratic Mutual Information estimation of mouse dLGN receptive fields reveals asymmetry between $\mathrm{ON}$ and $\mathrm{OFF}$ visual pathways.
}

\author{
Zhiguang $\mathrm{Mu}^{1}$, Konstantin Nikolic ${ }^{2}$ and Simon R. Schultz ${ }^{3}$
}

\begin{abstract}
The longstanding theory of "parallel processing" predicts that, except for a sign reversal, ON and OFF cells are driven by a similar pre-synaptic circuit and have similar visual field coverage, direction/orientation selectivity, visual acuity and other functional properties. However, recent experimental data challenges this view. Here we present an information theory based receptive field (RF) estimation method - quadratic mutual information (QMI) - applied to multi-electrode array electrophysiological recordings from the mouse dorsal lateral geniculate nucleus (dLGN). This estimation method provides more accurate RF estimates than the commonly used SpikeTriggered Average (STA) method, particularly in the presence of spatially correlated inputs. This improved efficiency allowed a larger number of RFs (285 vs 189 cells) to be extracted from a previously published dataset. Fitting a spatial-temporal Difference-of-Gaussians (ST-DoG) model to the RFs revealed that while the structural RF properties of $O N$ and OFF cells are largely symmetric, there were some asymmetries apparent in the functional properties of $\mathrm{ON}$ and $\mathrm{OFF}$ visual processing streams - with OFF cells preferring higher spatial and temporal frequencies on average, and showing a greater degree of orientation selectivity.
\end{abstract}

\section{INTRODUCTION}

Consider two image patches of identical size but opposite contrast. A light patch on a dark background is perceived differently to a dark patch on a light background: Helmholtz termed this illusion the "irradiation illusion" [1]. It arises partly because of the scattering effects of light, however Galilei suggested that this may also indicate a difference in spatial resolution [2]. Schiller and colleagues [3] found that 2-amino-4-phosphonobutyrate (APB) abolishes ON response in retinal ganglion cells (RGCs), the LGN and visual cortex, but has no effect on the center surround antagonism of OFF cells or the orientation and direction selectivity in the cortex. These and related findings suggest that the $\mathrm{ON}$ and OFF pathways remain largely separate though the LGN and cortex [4], opening up the potential for the separate pathways to be differently adapted to specific information processing demands.

Asymmetry between ON and OFF blue sensitivity-cones of retina was observed as early as in the 70s by de Monasterio [5]. Other asymmetric properties such as the size of the

\footnotetext{
${ }^{1}$ Neural Computation Unit, Okinawa Institute of Science and Technology, Okinawa, Japan zhiguang.mu@oist.jp

${ }^{2 a}$ School of Computing and Engineering, University of West London, London konstantin.nikolic@uwl.ac.uk

${ }^{2 b}$ Department of Electrical and Electronic Engineering, Imperial College London, London k.nikoliceimperial.ac.uk

${ }^{3}$ Department of Bioengineering and Centre for Neurotechnology, Imperial College London, London s.schultz@imperial.ac.uk

The authors would like to acknowledge this work is supported by EPSRC grant no. EP/N002474/1
}

receptive field of ON and OFF visual pathway have been observed in drosophila [6], rodents [7], tree shrews [8], leporids [9], cats [10] and primates [11], either in retina or visual cortex. The fact that this feature commonly existed among a diverse variety of species illustrates it may carry great importance in the functional role as a result of early development in the evolution [12]. In addition, the asymmetry in ON and OFF visual pathway has been linked with their physical properties, functions and information processing capabilities, this includes quantity [13], morphology \& receptive field size [14], contrast sensitivity [15], direction tuning [9], motion detection [6], depth perception [4] and velocity estimation [16].

Teasing apart potential information processing asymmetries between ON and OFF receptive fields requires, as a first step, accurate estimation of RF structure. In many previous studies, spike-triggered average (STA) estimates have typically been used. In many cases, the statistical inefficiency of this approach has required averaging across time to produce a purely spatial RF; as well as resulting in a decreased number of apparently responsive units (in cases where there are temporally separated ON and OFF components that nearly average out over time), it can disguise ON-OFF asymmetries. There is thus the need for improved RF estimators. For a linear RF model with Gaussian noise, minimising the mean squared error corresponds to a maximum likelihood estimation (MLE) or a maximum a posterior estimation (MAP) with uniform prior. If in addition, the stimulus is spatialtemporally decorrelated with zero mean, then this estimation is precisely equivalent to the STA method. However, for nonGaussian noise or spatial-temporally correlated input, STA provides biased and inaccurate estimates of RF structure. Here, following [17], we estimate RFs by minimising the quadratic approximation to the mutual information (QMI), without the Gaussian assumption, to provide a more accurate estimation of the mean of the receptive field parameters.

To the resulting RF estimates we further fitted a spatialtemporal Difference-of-Gaussian (ST-DoG) model to the receptive fields estimated from QMI method. This process reduced the dimension of the receptive field parameters down to several spatial-temporal Gaussian components, for further analysis. Observing a bimodal distribution of an ON-OFF index parameter, we classified neurons into two ON and OFF classes, based on this property. This procedure revealed an asymmetries in several properties between the ON and OFF visual pathways. 


\section{MATERIALS AND METHODS}

\section{A. Experimental Methods}

Experimental methods are described in detail in [18]. Briefly, mice (2-4 month old C57BL/6) were sedated with $0.5 \mathrm{mg} / \mathrm{kg}$ chlorprothixene and anaesthetised using 1-1.5\% isoflurane in $\mathrm{O}_{2}$. Multi-electrode arrays (MEAs, Neuronexus A1x16-Poly2-5mm-50s-177-A1) were vertically inserted into the brain until robust visual responses to gratings or white/black squares at a depth of 2500-3200 $\mu \mathrm{m}$ below the pial surface indicated that the dLGN had been found. Electrodes were coated in DiI (20 mg in $300 \mu \mathrm{l} \mathrm{DMSO}$ ) and recording locations verified with post-hoc histology. Visual stimuli were presented using custom-modified MATLAB scripts based on Psychtoolbox [19] for MATLAB (MathWorks). Several types of visual stimuli were presented, as described in [18]: contrast-modulated noise movies for RF mapping [20], as well as drifting grating stimuli of varying spatial frequency, temporal frequency, contrast and direction. Spike sorting was performed; basic analyses of this dataset are described in [18].

\section{B. Quadratic Mutual Information}

The idea of determining the receptive field by maximising the mutual information between stimulus and response (spike train) was popularised by Sharpee [21], as this allows the receptive field to be estimated using a spatially and/or temporally correlated stimulus. Quadratic Mutual Information (QMI) was proposed by Kapur as an alternative to the full mutual information measure [22]. The use of QMI for dimensionality reduction was proposed by Torkkola et al. [23], as this is computationally cheaper to use as the gain (or inverse cost) function. These two ideas were recently combined for receptive field extraction [17]. The mutual information between two continuous random variables $S$ and $R$ can be written as

$$
I(S ; R)=\iint p(s, r) \log \frac{p(s, r)}{p(s) p(r)} d r d s,
$$

where, for instance, $S$ might describe the visual stimulus, and $R$ the neural response. In practice, as the stimulus space is extremely large, we might replace $S$ by some transformed variable $Y$. The mutual information can be approximated by the quadratic form of mutual information [22] as

$$
\begin{aligned}
I_{\text {quad }}(Y, R)= & \iint(p(y, r)-p(y) p(r))^{2} d r d y \\
= & \iint p(y, r)^{2}+p(y)^{2} p(r)^{2} \\
& \quad-2 p(y, r) p(y) p(r) d r d y
\end{aligned}
$$

This latter quantity is much easier for calculation when the probability distributions are constructed from the data using kernel density estimation and the Gaussian kernel, because the integrals can be calculated in analytical form. The optimisation process:

$$
\mathbf{W}=\operatorname{argmax}_{\mathbf{W}}\left[I_{\text {quad }}(\mathbf{Y}, \mathbf{R})\right], \mathbf{Y}=\mathbf{W}^{\mathbf{T}} \mathbf{X}
$$

is unaffected, since the objective is to find the transformation W such that the mutual information between transformed inputs $\mathbf{Y}$ and weighted class labels $\mathbf{R}$ is maximised. For example the video input $(\mathrm{S})$ is transformed into abstract lower dimensional feature (Y) and the quadratic mutual information between feature $(\mathrm{Y})$ and cell spiking $(\mathrm{R})$ is maximised.

Spike trains were binned by stimulus frames, with a duration of $50 \mathrm{~ms}$ (effective frame rate $20 \mathrm{~Hz}$, although the monitor refresh rate was $120 \mathrm{~Hz}$ ). The number of spikes within each frame bin is denoted by $\left\{r_{t}\right\}_{t=1: T}$. We extracted the aligned stimulus window $s_{(t-\tau: t)}$ for each bin, up to a total window length of $\tau$ frames. The filtered signal $\left\{y_{t}:=\left\langle W, s_{(t-\tau: t)}\right\rangle\right\}_{t=1: T}$ is used to calculate the QMI with $\left\{r_{t}\right\}_{t=1: T}$, where $W$ is the linear receptive field vector of the cell, and $\langle.,$.$\rangle is the inner product.$

The original quadratic mutual information method was implemented by Katz et al. [17] for CPU in Matlab, but takes a long time to run (30-45 minutes per cell on our computer). We thus modified the method to incorporate GPU acceleration (with CUDA support), significantly reducing the computation time per cell (to 30-45 seconds per cell). Because the QMI method involves the manual setting of a bandwidth parameter $b$, Katz et al iteratively searched by first increasing then decreasing the bandwidth to find the suitable bandwidth value. In addition, they used the Matlab function fminsearch to find the optimal step-wise learning rate $\gamma$ and then used this learning rate to update the receptive field vector $W$ for each bandwidth chosen. The pseudocode for the algorithm is shown below. We instead used fminucn with default quasi-newton optimization, finding a slight speed improvement. As bandwidth selection for kernel density estimation is an extensive research topic on its own, we do not go into detail here. Pseudocode for the algorithm is shown in Algorithm 1.

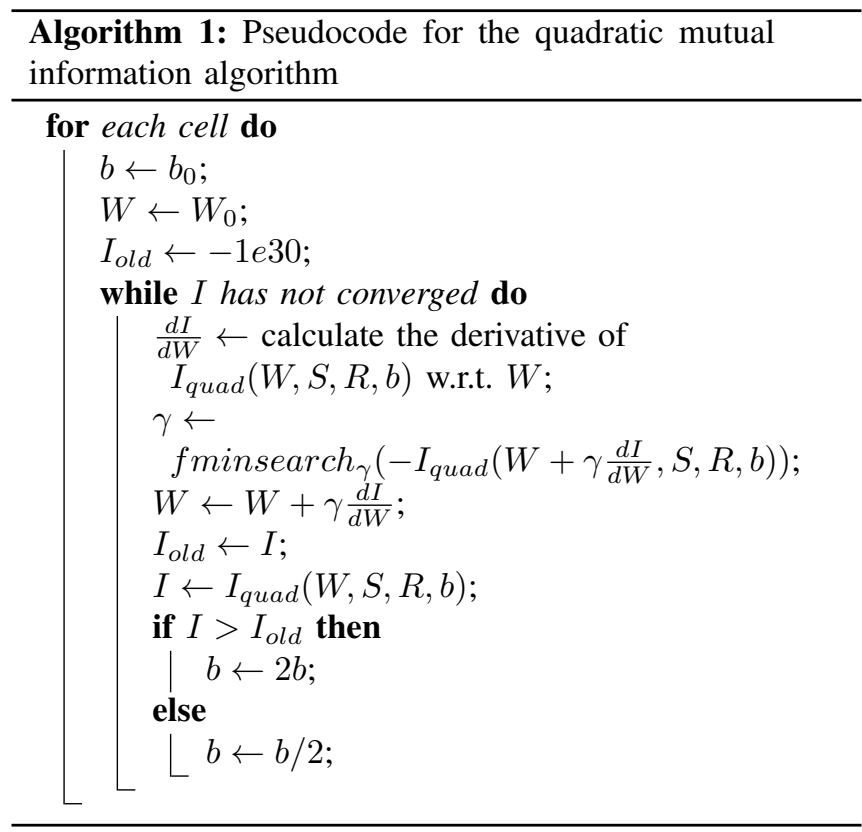




\section{C. $R F$ analysis with the Spatial-temporal Difference of Gaussians model}

We fit a spatial 2-dimensional and temporal 1-dimensional difference of Gaussians model to all receptive field vectors $W$ to reduce the number of parameters and make easier further clustering and analysis.

The original Difference-of-Gaussian (DoG) model consists of two spherical spatial Gaussian components with identical center position, whereas the amplitude of the two spatial Gaussian components follows reversal exponential. However, we found that the standard model cannot capture all spatiotemporal properties of the receptive field vectors we obtained from recordings from the mouse dLGN. In particular, many receptive fields estimated have a non-spherical shape, i.e. a non-diagonal sigma for spatial Gaussian components. In addition, two spatial Gaussian components often do not have the same peak center in our dataset. Finally, the model needed to be modified to allow for a larger magnitude (up to complete) temporal reversal in amplitude.

We have thus loosened the assumption and allowed the center and surround Gaussian component to have different peak position. The Gaussian component has also been modified to allow an elliptic spatial shape. In addition, instead of a weak reversal, we assumed a difference of two Gaussians for each amplitude in the temporal domain. The new model with 16 parameters, which we term the Spatial-Temporal Difference of Gaussians model (ST-DoG), is defined in full in the Appendix.

\section{ON-OFF Classification}

To classify cells into ON and OFF classes, we used the amplitudes of the ON and OFF components from the STDoG model fit, we define the ON-OFF index as:

$$
c_{O N-O F F}=\frac{\left|A_{+}\right|-\left|A_{-}\right|}{\left|A_{+}\right|+\left|A_{-}\right|}
$$

where $A_{+}$is the $\mathrm{ON}$ and $A_{-}$the OFF component amplitude parameter respectively. A value of $c_{O N-O F F}=1$ of this index corresponds to an ON-only cell, and $c_{O N-O F F}=-1$ corresponds to an OFF-only cell; cells with values lying in between these values possess both ON and OFF components. We use the value 0 as the decision boundary, i.e. if the ONOFF index is less than 0 , the cell is classified as an OFF cell. For example, in Fig 1 the $A_{+}$is the amplitude/peak of the yellow ST-Gaussian component, and similarly for $A_{-}$with blue ST-Gaussian component, for detail of those parameters, please refer to Appendix A.

\section{RESULTS}

First we analysed the performance of the QMI algorithm for mapping receptive fields on synthetic data, generated by simulating spike trains using a linear-nonlinear Poisson model, with linear filters derived from actual RFs observed in the dataset. An example is shown in Fig. 5a, depicting a "ground truth" RF over five $50 \mathrm{~ms}$ frames prior to spiking (spike occurrence in time at right of figure). In this example it is apparent that STA (Fig. 5b) somewhat over-estimates

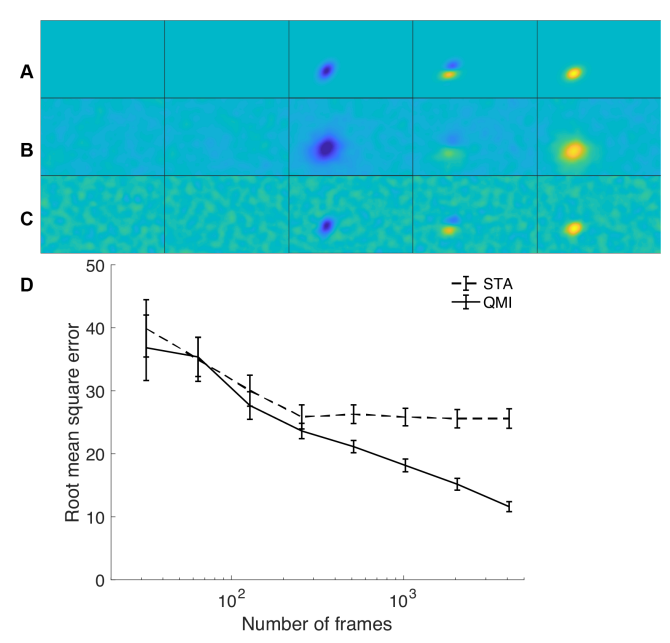

Fig. 1. Comparison of STA and QMI reconstructions for a simulated receptive field (RF). (a) "Ground truth" receptive field (constructed by us, but based on one of the cells in the dataset) used to simulate spike trains using a linear-nonlinear-Poisson neuron model. $50 \mathrm{~ms}$ frames shown across horizontally, of size $105 \times 65$ degrees. (b) Spike-triggered average (STA) reconstruction of the RF for 6000 frames. (c) QMI reconstruction of the RF for 6000 frames. (d) RMS error for increasing number of spikes used in the estimation (averaged over 10 different receptive fields) for STA and QMI RF estimates, 12 by 12 visual degree around the center of the RF was been used for calculation.

the size of the RF, which we assume is due to the biasing effect of spatial correlations in the contrast-modulated noise movie stimulus. In contrast, QMI obtains a much more accurate estimation of the receptive field (Fig. 5c), although apparently with the penalty of slightly more ripple in the baseline. This is an insubstantial issue, however, as the noise floor below a threshold can be trivially set to zero. QMI performance exceeds that of STA for datasets comprising 300 or more stimulus frames (Fig. 5d), or 15 seconds of data. For comparison, many minutes of data are normally collected in order to map RFs. In the original analysis of [18], 189 cells were assessed as both having good quality spike sorting and an adequate receptive field. By using QMI RF mapping, we were able to increase this to 285 . We believe that the reason for this is primarily that in [18] STA was used for RF mapping, with the consequence that it was necessary due to sampling limitations to average the RFs across time, i.e. no attempt was made to extract the temporal component of the RF. While this produces an adequate spatial RF for cells that are strongly dominated by $\mathrm{ON}$ or OFF components, or whose ON and OFF components are largely spatially nonoverlapping, it appears that there are a significant proportion of cells in the mouse dLGN with spatially overlapping ON and OFF components that come close to cancelling out if integrated over time to produce a single spatial RF. Several examples can be seen in Fig. 2. Of these cells, 133 were classified as ON cells and 152 as OFF cells, resulting in an overall ratio of ON to OFF cells of 46.6 to $53.4 \%$. Principal Component Analysis (Fig. 3) supported our conclusion that there were two broad categories of cells with differing properties, with nevertheless a region of parameter space where the two categories meet and are not (apart from the 


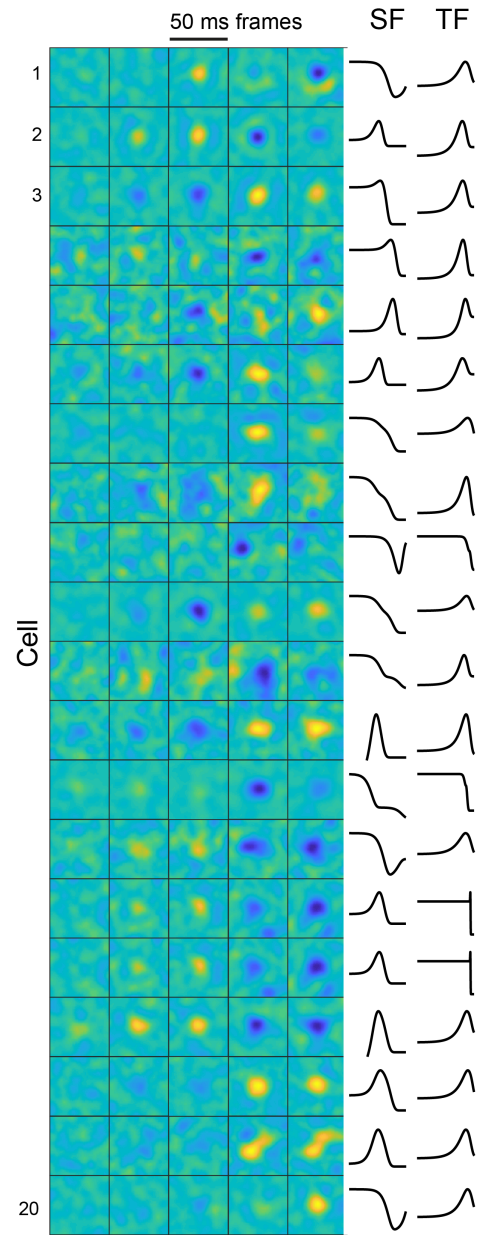

Fig. 2. Example RFs obtained by QMI optimization. Each square shows a $20 \times 20$ degree area centred around the RF; 5 temporal frames of the RF are shown, with the far right being the first prior to spike onset. At right are shown the spatial (SF) and temporal frequency (TF) tuning curves (fit as in [18]) for each of the 20 cells. SFs range logarithmically from 0.01 to $0.96 \mathrm{cyc} / \mathrm{deg}$, and TFs from 0.1 to $9.6 \mathrm{cyc} / \mathrm{sec}$.

sign of the ON-OFF index) distinguishable. We observed a clear bi-modal distribution of ON-OFF indices (Fig. 4a), despite the clearly continuous distribution ranging between -1 and 1.

We were able to extract numerous structural and functional properties of the RFs from the ST-DoG model. The effective RF radius, defined as the square root of the sum of the two "variances" of the Gaussian components $\sqrt{\sigma_{+}^{2}+\sigma_{-}^{2}}$ (see Appendix) did not differ substantially between $\mathrm{ON}$ and OFF cells (Fig. 4b). The ST-DoG model yields additional structural parameters, including the orientation of $\mathrm{ON}$ and OFF components of the receptive fields, shown for ON cells in Fig. 4c and OFF cells in Fig. 4d. There was no obvious asymmetry in these orientation parameters. Similarly, no difference between $\mathrm{ON}$ and OFF cells was apparent in the horizontal $\left(h_{+}-h_{-}\right)$or vertical $\left(v_{+}-v_{-}\right)$offset between the ON and OFF components of the ST-DoG model (Fig. 4e and f). An additional temporal structural parameter is the reversal time, or the time between the two temporal Gaussian peaks, $\tau_{+}-\tau_{-}$for ON cells and $\tau_{-}-\tau_{+}$for OFF cells(mean 43.768

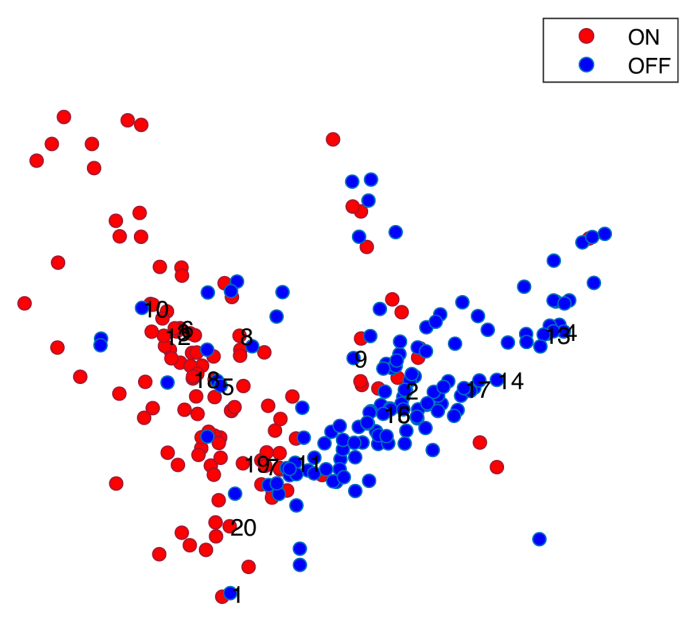

Fig. 3. Principal Component Analysis (PCA) of receptive field structure. PCA was performed to reduce the dimensionality of the description of the RF structure extracted from the ST-DoG model from 16 to 2 parameters for each cell. Numbers reflect cells shown in Fig. 2.

\pm 4.662 for OFF cells versus $33.415 \pm 5.727$ for ON cells; $\mathrm{p}=0.159$, two-sided t-test, $\mathrm{n}=133 \mathrm{ON}$ and $152 \mathrm{OFF}$ cells). ON cells showed on average a slightly (but not significantly) shorter latency, defined as $\min \left\{\tau_{+}, \tau_{-}\right\}$, the time to reach the first peak of either component, than OFF cells (mean $0.169 \pm 0.215$ (s.e.m.), $\mathrm{p}=0.182$, two-sided t-test, $\mathrm{n}=133 \mathrm{ON}$ and 152 OFF cells).

We were also able to relate the ST-DoG parameters to functional measurements obtained from tuning curve mapping experiments using drifting gratings. The Direction Selectivity Index (see [18], measured by $\sum F(\theta) e^{2 i \theta} / \sum F(\theta)$ where $F(\theta)$ gives the direction tuning curve) was similar for both ON and OFF cells ( $\mathrm{p}=0.681$, two-sided t-test, $\mathrm{n}=95$ and 98 respectively), Fig. 4i. This was not surprising given the relatively low representation of direction selective cells within this dataset. OFF cells, however, were significantly more orientation sensitive (Orientation Selectivity Index given by $\sum F(\theta) e^{i \theta} / \sum F(\theta)$; mean $0.1099 \pm 0.00882$ for OFF cells versus $0.0783 \pm 0.00583$ for $\mathrm{ON}$ cells; $\mathrm{p}=0.0036$, two-sided t-test, $\mathrm{n}=95$ and 98 ). OFF cells were similarly on average selective for higher spatial frequencies (mean 0.0978 \pm 0.0107 for OFF cells versus $0.0653 \pm 0.0094$ for $\mathrm{ON}$ cells; $\mathrm{p}=0.0256$, two-sided t-test, $\mathrm{n}=142$ OFF cells and 130 ON cells). OFF cells were also sensitive to slightly higher temporal frequencies (mean $3.683 \pm 0.1389$ for OFF cells versus $3.342 \pm 0.110$ for $\mathrm{ON}$ cells; $\mathrm{p}=0.0644$, two-sided t-test, $n=140$ OFF cells and 124 ON cells). Sample sizes differ in the above analyses because of the presence of lowpass tuned cells for whom a preferred spatial or temporal frequency was not defined.

\section{DISCUSSION}

We used quadratic mutual information to map receptive fields in the mouse dLGN. This yielded accurate, minimally biased estimates of RFs with substantially fewer samples than required by the method in most common use, the 

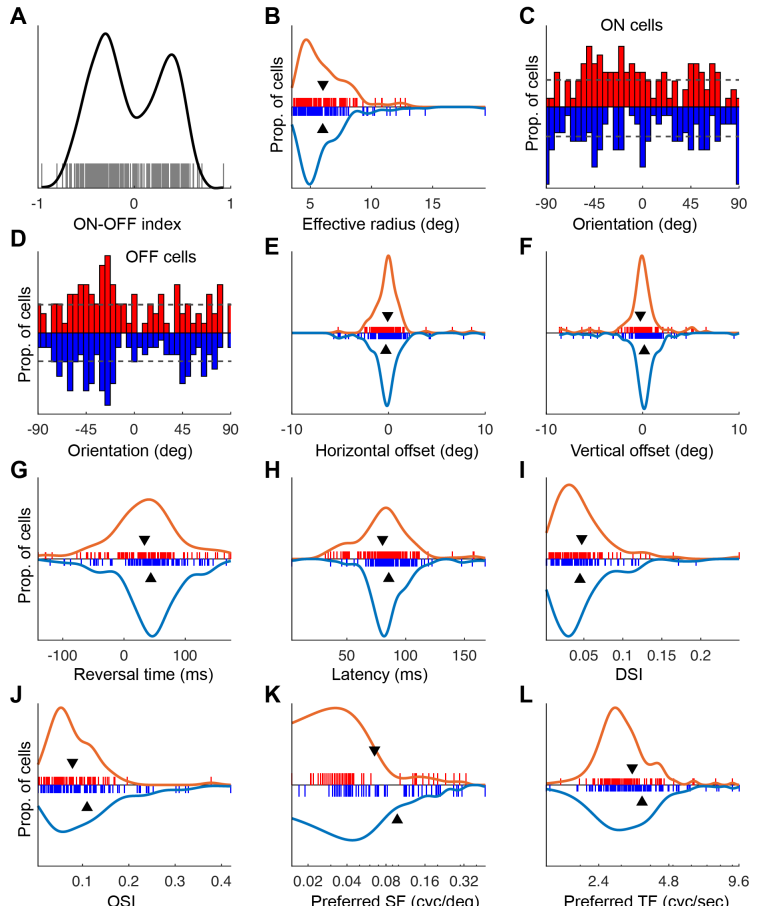

Fig. 4. Analysis of the functional properties of dLGN ON and OFF receptive fields (a) The distribution of ON-OFF indices for all 285 cells. (b) Effective radius for ON (red) and OFF (blue) cells. (c) Orientation (from $-\pi / 2$ to $\pi / 2$ ) of the ON component (red) and OFF component (blue) of the RF, for ON cells only. (d) As in (c), but for OFF cells. (e,f) Horizontal and vertical offset of ON and OFF RF components respectively, for ON cells (red) and OFF cells (blue). (g, h) Reversal time and latency for ON (red) and OFF cells (blue). (i) Direction selectivity Index (DSI). (j) Orientation Selectivity Index (OSI). (k) Preferred spatial frequency. (l) Preferred temporal frequency.

spike-triggered average. While it is well appreciated that the use of natural movie stimuli for receptive field mapping using spike-triggered approaches will result in biased RF estimates without the application of a correction procedure [24], such corrections can amplify noise and thus have adverse effects [25]. Information maximisation methods [21], [26] provide a much better approach for real-world stimuli, however the Kullback-Leibler mutual information is difficult to calculate and optimise, particularly for limited samples, meaning that approximations must usually be made. One such approximation, used in [21], is the use of the first order approximation to the information per spike [27]; this assumes independence of spikes, which is valid for e.g. the Poisson simulation we have used for validation, but does not hold for real world spike trains. An alternative approach, which we use here, is to use the Quadratic Mutual Information approximation [22]. As well as being easy to compute, this is differentiable, making it amenable to gradient-based optimization. In addition to advantages with respect to bias, QMI mapping of RFs is substantially more statistically efficient than STA mapping, requiring less recording duration to obtain RF structure.

QMI RF mapping yielded substantially more information about RF structure on an existing dataset [18], allowing the RFs of more cells to be obtained (285 as opposed to
189), and providing space-time RFs rather than purely spatial RFs that averaged over time. This allowed additional insight to be gained, and the enhanced dataset, while essentially yielding a picture of structural symmetry between $\mathrm{ON}$ and OFF receptive fields, did reveal asymmetries in a number of functional properties between the $\mathrm{ON}$ and OFF pathways in the mouse dLGN, including the orientation selectivity index and preferred spatial frequency (with a weaker effect apparent for the preferred temporal frequency). This implies some degree of functional specialisation of the $\mathrm{ON}$ and $\mathrm{OFF}$ pathways. While the role of this pathway splitting remains speculative (see [28] for a general discussion), one reason may relate to differences in uncertainty in dark versus wellilluminated areas of a visual scene.

One limitation of the dataset analysed was the use of a 20 $\mathrm{Hz}$ refresh rate for updating frames. This limited the temporal fidelity with which temporal aspects of RF structure can be extracted. Given that the QMI method enables statistically efficient extraction of spatiotemporal RF structure with high temporal fidelity, it will be of interest to collect additional experimental data with higher stimulus refresh rates. Analysis of finer temporal structure of the spatiotemporal RFs of dLGN neurons may reveal additional diversity in information processing functionality [29]. In addition, further analysis can be done by comparing this with other methods such as variants of spike-triggered covariance for highly correlated stimulus. However, we do note the high dimensional visual stimulus can make the covariance calculation computational expensive.

We finally remark that, although here, the QMI receptive field mapping method has been applied to an electrophysiological dataset, it is likely to have wider application. In particular, we expect it to find use in mapping sensory receptive fields through technology such as multiphoton calcium [30], [31] or voltage [32] imaging, and in additional characterisation domains such as mapping selectivity functions in spatial memory [33]. As well as for other mammals with higher visual acuity.

\section{REFERENCES}

[1] H. von Helmholtz, "Handbuch der physiologischen optik / Handbook of Physiological Optics (J. P. C. Southall, Trans. 3rd ed.)," Allgemeine Encyklopadie der Physik Vol 2, ed Karsten G(Voss, Leipzig, Germany)/Dover, pp. 186-193, 1867/(1910, 1962).

[2] G. Galilei, "Dialogue concerning the two chief world systems," Battista Landini, Florence, Italy, 1632.

[3] P. H. Schiller, "Central connections of the retinal on and off pathways," Nature, vol. 297, pp. 580-583, 1982.

[4] P. H. Schiller, J. H. Sandell, and J. H. R. Maunsell, "Functions of the on and off channels of the visual system," Nature, vol. 322, pp. 824-825, 1986.

[5] F. M. de Monasterio, "Asymmetry of on- and off-pathways of bluesensitive cones of the retina of macaques," Brain Research, vol. 166 no. 1, pp. 39-48, 1979.

[6] J. Haag, A. Mishra, and A. Borst, "A common directional tuning mechanism of Drosophila motion-sensing neurons in the on and in the off pathway," eLife, p. e29044, 2017.

[7] K. A. Zaghloul, K. Boahen, and J. B. Demb, "Different circuits for on and off retinal ganglion cells cause different contrast sensitivities," Journal of Neuroscience, vol. 23, no. 7, pp. 2645-2654, 2003. 
[8] J. Veit, A. Bhattacharyya, R. Kretz, and G. Rainer, "On the relation between receptive field structure and stimulus selectivity in the tree shrew primary visual cortex," Cereb. Cortex, vol. 24, no. 10, pp. 27612771, 2014.

[9] D. I Vaney, B. Sivyer, and W. Taylor, "Direction selectivity in the retina: Symmetry and asymmetry in structure and function," Nature Reviews Neuroscience, vol. 13, pp. 194-208, 022012.

[10] J. Z. Jin, C. Weng, C.-I. Yeh, J. A. Gordon, E. S. Ruthazer, M. P. Stryker, H. A. Swadlow, and J.-M. Alonso, "On and off domains of geniculate afferents in cat primary visual cortex," Nat. Neurosci., vol. 11, no. 1, p. 88, Dec 2007.

[11] D. Xing, C.-I. Yeh, J. Gordon, and R. M. Shapley, "Cortical brightness adaptation when darkness and brightness produce different dynamical states in the visual cortex," Proc. Natl. Acad. Sci. U.S.A., vol. 111, no. 3, pp. 1210-1215, Jan 2014.

[12] D. A. Clark, J. E. Fitzgerald, J. M. Ales, D. M. Gohl, M. A. Silies, A. M. Norcia, and T. R. Clandinin, "Flies and humans share a motion estimation strategy that exploits natural scene statistics," Nature Neuroscience, vol. 17, no. 2, pp. 296-303, 2014.

[13] K. M. Ahmad, K. Klug, S. Herr, P. Sterling, and S. Schein, "Cell density ratios in a foveal patch in macaque retina," Visual Neuroscience, vol. 20, no. 2, p. 189, 2003.

[14] S. Ravi, D. Ahn, M. Greschner, E. Chichilnisky, and G. D. Field, "Pathway-specific asymmetries between on and off visual signals," Journal of Neuroscience, vol. 38, no. 45, pp. 9728-9740, 2018.

[15] V. Zemon, J. Gordon, and J. Welch, "Asymmetries in on and off visual pathways of humans revealed using contrast-evoked cortical potentials," Visual Neuroscience, vol. 1, no. 1, p. 145-150, 1988.

[16] A. Leonhardt, G. Ammer, M. Meier, E. Serbe, A. Bahl, and A. Borst, "Asymmetry of drosophila on and off motion detectors enhances realworld velocity estimation," Nature Neuroscience, vol. 19, 022016.

[17] M. L. Katz, T. J. Viney, and K. Nikolic, "Receptive field vectors of genetically-identified retinal ganglion cells reveal cell-type-dependent visual functions," PLOS ONE, vol. 11, no. 2, pp. 1-29, 2016.

[18] J. Tang, S. C. Ardila Jimenez, S. Chakraborty, and S. R. Schultz, "Visual receptive field properties of neurons in the mouse lateral geniculate nucleus," PLOS ONE, vol. 11, no. 1, pp. 1-34, 012016.

[19] M. Kleiner, D. Brainard, and D. Pelli, "What's new in Psychtoolbox3?" Perception, vol. 36 EVCP Abstract Supplement, 2007.

[20] C. M. Niell and M. P. Stryker, "Highly selective receptive fields in mouse visual cortex," Journal of Neuroscience, vol. 28, no. 30, pp. 7520-7536, 2008.

[21] T. O. Sharpee, "Neural Responses To Natural Signals: Maximally Informative Dimensions," Neural Computation, vol. 16, pp. 223-250, 2004.

[22] J. Kapur, Measures of Information and Their Applications. Wiley, 1994.

[23] K. Torkkola, "Feature extraction by non-parametric mutual information maximization," Journal of Machine Learning Research, vol. 3, pp. 1415-1438, 2003.

[24] J. Touryan, G. Felsen, and Y. Dan, "Spatial structure of complex cell receptive fields measured with natural images," Neuron, vol. 45, no. 5, pp. 781-791, 2005.

[25] F. E. Theunissen, K. Sen, and A. J. Doupe, "Spectral-temporal receptive fields of nonlinear auditory neurons obtained using natural sounds," Journal of Neuroscience, vol. 20, no. 6, pp. 2315-2331, 2000.

[26] T. O. Sharpee, "Comparison of information and variance maximization strategies for characterizing neural feature selectivity," Statistics in medicine, vol. 26, no. 21, pp. 4009-4031, 2007.

[27] W. Skaggs, B. Mcnaughton, and K. Gothard, "An information-theoretic approach to deciphering the hippocampal code," Advances in neural information processing systems, vol. 5, pp. 1030-1037, 1992.

[28] J. Gjorgjieva, H. Sompolinsky, and M. Meister, "Benefits of pathway splitting in sensory coding," Journal of Neuroscience, vol. 34, no. 36, pp. 12 127-12 144, 2014.

[29] D. M. Piscopo, R. N. El-Danaf, A. D. Huberman, and C. M. Niell, "Diverse Visual Features Encoded in Mouse Lateral Geniculate Nucleus," Journal of Neuroscience, vol. 33, no. 11, pp. 4642-4656, 2013.

[30] S. L. Smith and M. Häusser, "Parallel processing of visual space by neighboring neurons in mouse visual cortex," Nature neuroscience, vol. 13, no. 9, p. 1144, 2010.

[31] S. R. Schultz, C. S. Copeland, A. J. Foust, P. Quicke, and R. Schuck, "Advances in two-photon scanning and scanless microscopy technologies for functional neural circuit imaging," Proceedings of the IEEE, vol. 105, no. 1, pp. 139-157, 2016.
[32] P. Quicke, C. L. Howe, P. Song, H. V. Jadan, C. Song, T. Knöpfel, M. Neil, P. L. Dragotti, S. R. Schultz, and A. J. Foust, "Subcellular resolution three-dimensional light-field imaging with genetically encoded voltage indicators," Neurophotonics, vol. 7, no. 3, p. 035006 , 2020

[33] M. A. Go, J. Rogers, G. P. Gava, C. Davey, S. Prado, Y. Liu, and S. R. Schultz, "Place cells in head-fixed mice navigating a floating real-world environment," bioRxiv, 2020.

[34] P. Dayan and L. F. Abbott, Theoretical Neuroscience: Computational and Mathematical Modeling of Neural Systems. The MIT Press, 2005.

\section{APPENDIX}

\section{A. Spatial-temporal Difference of Gaussian (ST-DoG)}

We define our new model with the following parameters: $h_{+}-$the horizontal position of the positive Gaussian components, $v_{+}$- the vertical position of the positive Gaussian components, $h_{-}-$the horizontal position of the negative Gaussian components, $v_{-}$- the vertical position of the negative Gaussian components, $\sigma_{h+}, \sigma_{v+}, \theta_{+}-$eclipse radius and angle of the positive spatial Gaussian component, $\sigma_{h-}, \sigma_{v-}, \theta_{-}-$eclipse radius and angle of the negative spatial Gaussian component, $A_{+}, \tau_{+}, \sigma_{+}-$the temporal positive Gaussian component, $A_{-}, \tau_{-}, \sigma_{-}-$the temporal negative Gaussian component. We first define:

$$
\begin{gathered}
\mu_{+}=\left(\begin{array}{c}
h_{+} \\
v_{+}
\end{array}\right) p=\left(\begin{array}{l}
h \\
v
\end{array}\right) \\
\Sigma_{+}=\left(\begin{array}{cc}
\cos \theta_{+} & -\sin \theta_{+} \\
\sin \theta_{+} & \cos \theta_{+}
\end{array}\right)\left(\begin{array}{cc}
\sigma_{h+} & 0 \\
0 & \sigma_{v+}
\end{array}\right)
\end{gathered}
$$

And similarly for $\mu_{-}$and $\Sigma_{-}$. The receptive field is then defined with those parameters by:

$$
\begin{aligned}
W(h, v, \tau) & =A_{O N}(\tau) \exp \left[-\frac{1}{2}\left(p-\mu_{+}\right) \Sigma_{+}\left(p-\mu_{+}\right)\right] \\
& -A_{O F F}(\tau) \exp \left[-\frac{1}{2}\left(p-\mu_{-}\right) \Sigma_{-}\left(p-\mu_{-}\right)\right]
\end{aligned}
$$

Amplitude for ON components (similar for OFF):

$$
A_{O N}(\tau)=A_{+} \exp \left[-\frac{\left(\tau-\tau_{+}\right)^{2}}{2 \sigma_{+}^{2}}\right]
$$

Note we restricted $A_{+}$and $A_{-}$to be positive and $\left|\tau_{+}-\tau_{-}\right|>$ $25 \mathrm{~ms}$ so that they will not cancel out in temporal domain to cancel each other out, causing overfitting. We used a global optimisation algorithm (adaptive mesh search) to find the optimal parameters, which is implemented in Matlab as the function fmincon.

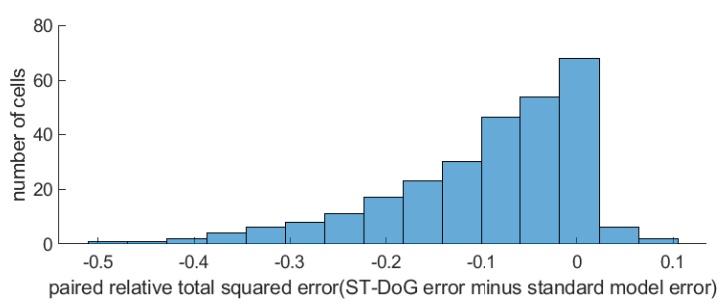

Fig. 5. Comparison of ST-DoG and standard model from [34]. Paired total squared error difference has been plotted. 